\title{
Acute Bacterial Skin and Skin Structure Infections Treated with Intravenous Antibiotics in the Emergency Department or Observational Unit: Experience at the Detroit Medical Center
}

\author{
Kimberly C. Claeys · Abdalhamid M. Lagnf · Trishna B. Patel • \\ Manu G. Jacob · Susan L. Davis · Michael J. Rybak \\ To view enhanced content go to www.infectiousdiseases-open.com \\ Received: April 2, 2015 / Published online: June 9, 2015 \\ (C) The Author(s) 2015. This article is published with open access at Springerlink.com
}

\section{ABSTRACT}

Introduction: Acute bacterial skin and skin structure infections (ABSSSIs) are frequently treated in emergency departments (EDs) or observation units (OUs) initially with intravenous (IV) antibiotics before discharge on oral therapy. This study aims to describe

Prior Presentations: Interscience Conference on Antimicrobial Agents and Chemotherapy, Washington DC, September 2014.

Electronic supplementary material The online version of this article (doi:10.1007/s40121-015-0069-7) contains supplementary material, which is available to authorized users.

K. C. Claeys - A. M. Lagnf · M. J. Rybak Anti-Infective Research Laboratory, Eugene Applebaum College of Pharmacy and Health Sciences, Wayne State University, Detroit, MI 48201, USA

K. C. Claeys · A. M. Lagnf - T. B. Patel

M. G. Jacob · S. L. Davis · M. J. Rybak $(\bowtie)$ Department of Pharmacy Practice, Eugene Applebaum College of Pharmacy and Health Sciences, Wayne State University, Detroit, MI 48201, USA

e-mail:m.rybak@wayne.edu

\section{S. L. Davis}

Department of Pharmacy Practice, Henry Ford Hospital, 2799 W Grand Blvd, Detroit, MI 48202, USA
ABSSSI patients discharged directly from EDs/ OUs.

Methods: This is a retrospective cohort study of patients with ABSSSIs treated in EDs/OUs of the Detroit Medical Center from 2012 to 2014. Adults with less than $24 \mathrm{~h}$ of IV antibiotics without hospital admission were included. Demographics, clinical characteristics, and severity were compared between ED and OU patients. Resource utilization, including tissue and blood cultures, and use of radiographic analysis was also collected. The primary outcome was 96-h ED revisit/hospitalization.

Results: Analysis included 308 patients; 219 ED and 89 OU. OU patients were significantly more likely to be obese, have COPD/asthma, be diagnosed with cellulitis, and meet at least one systemic inflammatory response syndrome (SIRS) criterion. Tissue cultures were obtained in $21.7 \%$ of abscesses in the ED; $67.9 \%$ were in uncomplicated abscesses. In the OU tissue cultures were obtained in $48.8 \%$ of abscesses and $37.5 \%$ were uncomplicated cases. Blood cultures were drawn in $18.3 \%$ of ED patients and $56.2 \%$ of OU patients, not significantly associated with the presence of SIRS criteria. Radiology was used in the diagnosis of ABSSSIs 
in $33.5 \%$ of $\mathrm{ED}$ versus $69.5 \%$ OU patients $(p<0.001)$, Plain film radiograph being the most common. Thirty patients revisited the ED or required hospitalization within 96 h, 23 from the $\mathrm{ED}(p=0.479)$. Prior history of ABSSSI (adjusted odds ratio $[\mathrm{aOR}]=2.382, \quad 95 \%$ confidence interval [CI] 1.264-6.346) and location on torso/buttocks $(\mathrm{aOR}=2.355,95 \%$ CI 1.067-5.197) were independent predictors.

Conclusions: The low rate of ED revisit/ hospitalization supports the use of OUs for low acuity ABSSSIs requiring initial IV therapy. Resource utilization within EDs/OUs for the management of ABSSSIs needs to be evaluated for unnecessary testing/procures.

Keywords: Acute bacterial skin and skin structure infections; Emergency department; Intravenous antibiotics; Observation unit

\section{INTRODUCTION}

Acute bacterial skin and skin structure infections (ABSSSIs) are among the most common infections encountered in both community and hospital settings [1, 2]. According to the 2010 National Hospital Ambulatory Medical Care Survey (NHAMCS), diseases of the skin and subcutaneous tissue account for $4.2 \%$ of all emergency department (ED) visits in the United States [3]. Additionally, national trends indicate that the rate of ABSSSIs has increased by approximately 50\% since 1997, with the majority of patients being treated and released directly from the $\mathrm{ED}[4,5]$. There is a wide clinical spectrum of ABSSSIs, from simple uncomplicated abscesses to life-threatening necrotizing fasciitis, and agreement upon severity classification is lacking [6]. Most ABSSSIs, aside from gangrene and fasciitis, are treated on an outpatient basis. Although the majority of patients receive care in the ED and are not admitted, complications due to ABSSSIs often lead to hospitalization. It is not presently known how many revisits/admissions are represented among the over 600,000 to 800,000 documented admissions for ABSSSIs in the U.S. annually [2]. Two Canadian studies have reported outpatient failure rates, resulting in subsequent admission, of approximately $20-25 \%$ for cellulitis exclusively treated in the ED $[7,8]$.

In a study by Edelsberg and colleagues, antibiotic clinical failure rates were documented for over 10,000 hospitalized patients, $22.8 \%$ of all patients studied [2]. This was mirrored by Berger and colleagues followup study, which demonstrated failure rates of 16.6-34.1\% [9]. These results demonstrated that patients who initially fail empiric treatment often require prolonged hospital stays and intravenous (IV) antibiotics. Alternatively, some patients are observed in the hospital for short periods of time, either as brief admissions or in observation units (OUs). According to the American College of Emergency Physicians (ACEP), $70 \%$ of patients selected from treatment in OUs should be discharged before $24 \mathrm{~h}$ of care [10]. The use of these OUs allows for hospital avoidance, decreased resource utilization, and decreased exposure to the healthcare environment, and thus a decrease in adverse events. The use of observation status has increased by $26 \%$ among Medicare beneficiaries since 2008 [11]. Despite the high incidence of ABSSSIs treated without hospital admission, data examining the burden of illness, processes of care, and decisions used to determine care are lacking [12-14]. In addition, many professional societies, including ACEP, are advocating for more evidence-based, conscientious use of diagnostic resources to optimize care and decrease waste within 
healthcare [15]. The objectives of this study were to determine the burden of illness and services received for patients with less than $24 \mathrm{~h}$ of IV therapy for ABSSSIs before being discharged directly from EDs and OUs.

\section{METHODS}

\section{Study Design}

This retrospective observational study was completed at the Detroit Medical Center (DMC) between April 2012 and September 2014. Institutional Review Board approval was obtained from Wayne State University with a waiver of informed consent.

\section{Study Setting and Population}

The study was conducted at the DMC. The DMC consists of nine hospitals and is the largest healthcare provider for Southeast Michigan. The study was completed at two acute care trauma institutions (Detroit Receiving Hospital and Harper University Hospital) and two community-based hospitals (Sinai-Grace Hospital and Huron Valley Sinai Hospital) within the DMC.

\section{Study Protocol}

Patients were screened consecutively using a standardized approach throughout the study period and data was collected retrospectively, after completion of care. Eligible patients were those between 18 and 89 years of age diagnosed with ABSSSI per the treating physician and presenting with at least three of the following local signs/symptoms: pain, tenderness, swelling erythema, warmth, drainage/ discharge, induration, and/or lymph node swelling/tenderness.
Patients were excluded from analysis if osteomyelitis/septic arthritis, gas gangrene/ necrotizing infections were suspected. Additional exclusion criteria included the presence of prosthetic hardware or invasive devices suspected to be the source of infection but could not be removed, receiving oral antibiotics only, treatment with IV antibiotics for greater that $24 \mathrm{~h}$, odontogenic infections, open burn of greater than $30 \%$ of body surface area, pregnant or nursing, or prisoners. For patients with multiple eligible visits during the study period, only the first was included as the index visit. Patients were categorized as receiving care in the $\mathrm{ED}$ if all management for the index visit was received within the ED; patients were categorized as receiving care in the $\mathrm{OU}$ if they received any services in the $\mathrm{OU}$ prior to discharge home.

\section{Measures}

Patient-level data collected included demographics, comorbid conditions and Charlson Comorbidity Score, antibiotic history (last 90 days) and hospitalization history (last 180 days) as available, history of ABSSSI as available, type of ABSSSI per Food and Drug Administration definitions [16], complicated versus uncomplicated ABSSSI [1, 17], criteria for systemic inflammatory response syndrome (SIRS), empiric treatment, other therapeutic interventions for ABSSSI (debridement, incision and drainage, amputation), procedures and tests used to aid in the diagnosis of ABSSSI, discharge antibiotic prescription(s), type of insurance, time (in $h$ ) till discharge from ED or OU, and 96-h ED revisit/hospitalization. Two previously published severity-scoring systems that have been studied in the management of ABSSSIs were also calculated and compared: the Clinical 
Resource Efficiency Support Team (CREST)/Eron Classification and Standardized Early Warning Score (SEWS) (Fig. 1) [18, 19]. The primary outcome of interest was "early" ED revisit or hospital admission, noted to be within 96-h post-initial encounter [20].

\section{Data Analysis}

Descriptive statistics were determined for patient demographics, comorbid conditions, severity of illness per CREST/Eron and SEWS, services/diagnostic procedures received, and outpatient antibiotics prescribed. Characteristics were compared between ED and OU patients to determine possible differences in the treatment pattern. Student $t$ test, Mann-Whitney $U$ test, or Wilcoxon rank sum test was used for continuous and ordinal variables and Pearson's Chi-squared or Fisher's exact test was used for categorical variables. Correlations were determined through Spearman Rank-Order Correlation. A multivariable logistic regression analysis was conducted to determine independent risk factors for the primary outcome of interest. Variables significantly associated with the outcome on univariate analysis $(p \leq 0.1)$ or with clinical and scientific rationale were included in the explanatory model using backwards-stepwise logistic regression. Data analysis was conducted using SPSS Statistics (Version 22.0. Armonk, NY: IBM Corp.).

\begin{tabular}{|l|c|c|c|c|}
\hline \multicolumn{7}{|c|}{ CREST CLASSIFICATION } \\
\hline CREST I & No signs & None & Oral & Outpatient \\
\hline CREST II & $\begin{array}{c}\text { May or may not have } \\
\text { systemic illness } \\
\text { Inpatient }\end{array}$ \\
\hline CREST III & $\begin{array}{c}\text { Significant }- \text { confusion, } \\
\text { tachycardia, tachypnea, } \\
\text { hypotension }\end{array}$ & $\begin{array}{c}\text { Peripheral vascular, } \\
\text { obsity, venous } \\
\text { insufficiency }\end{array}$ & IV & $\begin{array}{c}\text { Hospital for } 48 \mathrm{~h} \\
\text { then outpatient }\end{array}$ \\
\hline CREST IV & Sepsis syndrome & Unstable & $\begin{array}{c}\text { IV +/- surgical } \\
\text { debridement }\end{array}$ & Hospital \\
\hline
\end{tabular}

\begin{tabular}{|c|c|c|c|c|}
\hline \multicolumn{5}{|c|}{ Standardized Early Warning System } \\
\hline Parameter & \multicolumn{4}{|c|}{ Score } \\
\hline & 3 & 2 & 1 & 0 \\
\hline Respiratory (breaths/m) & $\leq 8$ or $\geq 36$ & $31-35$ & $90-92$ & $9-20$ \\
\hline Oxygen saturation (\%) & $<85$ & $85-89$ & 80 & $36-37.9$ \\
\hline Temperature $\left({ }^{\circ} \mathbf{C}\right)$ & $<34$ & $34-34.9$ or $\geq 38.5$ & $35-35.9$ or $38-38.4$ & $36-99$ \\
\hline Systolic BP $(\mathbf{m m H g})$ & $\leq 69$ & $70-79$ or $\geq 200$ & $100-199$ \\
\hline Heart rate (bpm) & $\leq 29$ or $\geq 130$ & $30-39$ or $110-129$ & $40-49$ or $100-109$ & $50-99$ \\
\hline Responsiveness & Unresponsive & Painful stimuli & Verbal stimuli & Alert \\
\hline
\end{tabular}

Fig. 1 Clinical Resource Efficiency Support Team (CREST) Classification and Standardized Early Warning System (SEWS) Score (adapted from $[1,18,23]$ ) 
This was an institutional review board approved, retrospective, observational study at an urban, academic medical center. All procedures followed were in accordance with the ethical standards of the responsible committee on human experimentation (institutional and national) and with the Helsinki Declaration of 1975 , as revised in 2000. Informed consent was waived by Wayne State University institutional review board.

\section{RESULTS}

In total, 1877 patients' electronic medical records were reviewed for study inclusion, of these 1569 were excluded from the current study. Reasons for exclusion are detailed in Fig. 2. The remaining 308 were included in the final analysis, with $219(71.1 \%)$ treated in the ED and 89 (28.9\%) sent to the OU. Patient characteristics varied considerably according to setting of care (Table 1). Uncomplicated abscesses were often managed in the ED while

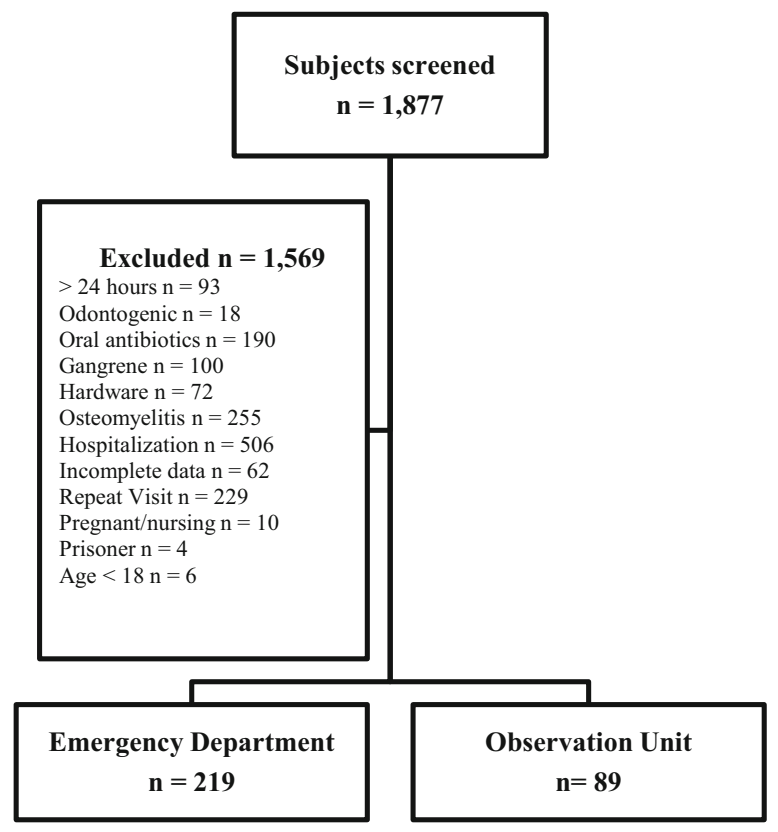

Fig. 2 Study Consolidated Standards of Reporting Trials (CONSORT) cellulitis was more commonly treated in the OU. Patients with comorbid conditions such as obesity (BMI $\geq 30 \mathrm{~kg} / \mathrm{m}^{2}$ ) or asthma/COPD were more likely to have a stay in the OU $(p=0.05$, $p=0.008$, respectively). Criteria for SIRS, except for temperature, were also significantly associated with OU treatment. Socioeconomic factors, such as insurance, had no significant impact on setting of care.

One hundred and six (80.4\%) ED patients and $66(74.2 \%)$ OU patients were determined to be CREST/Eron Class I, suggesting treatment with oral antibiotics on an outpatient basis would be appropriate. The remainder were Class II, indicating a short observation period with IV antibiotics is appropriate. There was no significant correlation between CREST/Eron Class and setting of care. The median (interquartile range) SEWS was $0(0-1)$ for ED patients and $1(0-1)$ for OU patients and this was significantly correlated with setting of care $(p=0.01)$. Patients with a SEWS score $\geq 1$ were almost twice as likely to be treated in the OU compared to the ED (odds ratio [OR] 1.96, 95\% confidence interval [CI] 1.17-4.13, $p=0.017$ ).

Not surprisingly, there were also considerable differences in the management and utilization of resources in patients treated in the ED compared to the OU, where patients received care for a longer period of time (median 20.56 versus $3.46 \mathrm{~h}$ ) and were overseen primarily by Internal Medicine physicians. An important difference was the collection of cultures from purulent lesions. Of the 129 ED patients with purulent lesions, 28 (21.7\%) had cultures obtained, $67.9 \%$ of these were collected from uncomplicated abscesses. In the OU cultures were obtained in 16 of 33 (48.8\%) of purulent lesions, with $37.5 \%$ from uncomplicated cases. Blood cultures were drawn from $40(18.3 \%)$ ED patients and 50 (56.2\%) OU patients. There was no association 
Table 1 Demographics by setting of care

\begin{tabular}{|c|c|c|c|}
\hline Characteristics & Emergency department $(n=219)$ & Observation unit $(n=89)$ & $p$ value \\
\hline Age, median (IRQ) & $41(28-50)$ & $46(33-55)$ & $0.083^{\mathrm{a}}$ \\
\hline Male sex & $121(55.3)$ & $42(47.2)$ & 0.143 \\
\hline Charlson score, median (IQR) & $1(0-2)$ & $1(0-2)$ & $0.539^{\mathrm{a}}$ \\
\hline \multicolumn{4}{|l|}{ Comorbidities } \\
\hline Diabetes mellitus & $31(14.2)$ & $19(21.3)$ & 0.121 \\
\hline Obesity $\left(\mathrm{BMI} \geq 30 \mathrm{~kg} / \mathrm{m}^{2}\right)$ & $23(10.5)$ & $17(19.1)$ & 0.042 \\
\hline Chronic kidney disease & $12(5.5)$ & $1(1.1)$ & $0.118^{\mathrm{b}}$ \\
\hline Liver disease & $5(2.3)$ & $5(5.6)$ & $0.159^{\mathrm{b}}$ \\
\hline COPD/asthma & $16(7.3)$ & $16(18.0)$ & 0.005 \\
\hline Injection drug use & $23(10.5)$ & $11(12.4)$ & 0.637 \\
\hline Peripheral vascular disease & $6(2.7)$ & $0(0.0)$ & $0.115^{\mathrm{b}}$ \\
\hline Prior MRSA infection & $4(1.8)$ & $3(3.4)$ & $0.410^{\mathrm{b}}$ \\
\hline Prior history of ABSSSI & $42(19.2)$ & $23(25.8)$ & 0.194 \\
\hline Prior hospitalization, 180 days & $15(6.8)$ & $10(11.2)$ & 0.201 \\
\hline Prior antibiotics, 90 days & $30(13.7)$ & $20(22.5)$ & 0.058 \\
\hline Primary location of ABSSSI & & & 0.004 \\
\hline Head/neck & $48(21.9)$ & $6(6.7)$ & 0.001 \\
\hline Hand & $18(8.2)$ & $13(14.6)$ & 0.091 \\
\hline Arm & $37(16.9)$ & $18(20.2)$ & 0.489 \\
\hline Leg & $35(16.0)$ & $26(29.2)$ & 0.008 \\
\hline Foot & $14(6.4)$ & $6(6.7)$ & 0.910 \\
\hline Trunk/buttocks & $65(29.7)$ & $20(22.5)$ & 0.200 \\
\hline \multicolumn{4}{|l|}{ SIRS at presentation } \\
\hline Fever $\left(\right.$ temperature $\geq 38.0^{\circ} \mathrm{C}$ ) & $46(21.0)$ & $23(25.8)$ & 0.356 \\
\hline WBC $>11,000$ or $<4000$ & $15(6.8)$ & $15(16.9)$ & 0.007 \\
\hline Heart rate $\geq 90$ beats $/ \mathrm{min}$ & $84(38.4)$ & $53(59.6)$ & 0.001 \\
\hline Respiratory rate $\geq 20 / \mathrm{min}$ & $5(2.3)$ & $9(10.1)$ & $0.003^{\mathrm{b}}$ \\
\hline \multicolumn{4}{|l|}{ Insurance type } \\
\hline Medicaid & $64(29.2)$ & $32(36.0)$ & 0.248 \\
\hline Medicare & $26(11.9)$ & $7(7.9)$ & 0.303 \\
\hline Private & $38(17.4)$ & $18(20.2)$ & 0.553 \\
\hline None & $81(37.0)$ & $23(25.8)$ & 0.061 \\
\hline
\end{tabular}

$A B S S S I$ acute bacterial skin and skin structure infection, $B M I$ body mass index, COPD chronic obstructive pulmonary disease, IQR interquartile range, MRSA methicillin-resistant Staphylococcus aureus, WBC white blood cell count

${ }^{a}$ Wilcoxon rank sum test

b Fisher's exact test 
between the presence of systemic toxicity (SIRS) or comorbid conditions and the use of blood cultures. Radiology was used to aid in the diagnosis of ABSSSIs in 69 (31.5\%) of ED and $59(66.3 \%)$ of OU patients $(p<0.001)$, the majority (89.5\%) through plain-film radiograph, followed by ultrasound $(7.5 \%)$ and CT (5.2\%). There was no significant difference in the use of radiology in complicated versus uncomplicated infections, either abscesses $(30.1 \%$ versus $32.3 \%)$ or cellulitis $(53.5 \%$ versus 63.4\%). Initial laboratory assessment was obtained in all patients; C-reactive protein and erythrocyte sedimentation rate were not routinely measured.

The most common empiric IV antibiotics were clindamycin, vancomycin, and ampicillin/sulbactam, respectively (Table 2). Patients with abscesses, both complicated and uncomplicated, were significantly more likely to receive clindamycin in the $\mathrm{ED}(p=0.001)$ while vancomycin was more common in uncomplicated abscesses and cellulitis in the OU ( $p=0.001$ and 0.033 , respectively). The most common discharge antibiotics were oral clindamycin and trimethoprim/ sulfamethoxazole (TMP/SMX) in both the ED and OU. More patients with complicated abscesses in the ED compared to OU received an agent providing empiric coverage for community acquired methicillin-resistant Staphylococcus aureus (CAMRSA) (TMP/SMX, clindamycin, and doxycycline $)(p=0.019)$. Among patients with cellulitis, 88 (71.0\%) were prescribed an agent active against CAMRSA. For patients with uncomplicated abscesses that underwent incision and drainage 64 (91.4\%) received a discharge antibiotic, primarily those active against CAMRSA.

Thirty patients $(9.7 \%)$ from the entire cohort, 23 (10.5\%) ED and 7 (7.9\%) OU experienced the primary endpoint of $96-\mathrm{h} \mathrm{ED}$ revisit or hospitalization. Among the entire cohort 30-day infection-related admission was low $(23,7.5 \%)$, with no significant difference between patients seen in the ED versus OU. Univariate analysis (Table 3) demonstrated that prior history of ABSSSI, prior history of MRSA, meeting SIRS criteria for alteration in body temperature, and ABSSSI located on the torso/ buttocks were risk factors for 96-h ED revisit. Due to the limited number of subjects experiencing the primary outcome, only these variables were tested in the regression model. Through multivariable backwards-logistic regression (Table 4), prior history of ABSSSI (adjusted odds ratio [aOR] $2.38 \quad[95 \%$ CI 1.26-6.35]) and location on the torso/buttocks (aOR $2.36 \quad[95 \%$ CI $1.07-5.20]$ ) were independently associated with 96-h ED revisit or hospitalization. Among the subset of patients managed only in the ED, prior history of ABSSSI (aOR 3.03 [95\% CI 1.19-7.80]) and meeting SIRS criteria for temperature (aOR 2.51 [95\% CI 1.00-6.35]) were independently associated with 96-h ED revisit or hospitalization.

\section{DISCUSSION}

The types of ABSSSIs seen in EDs and OUs are diverse and there is a high degree of variability in the management strategies employed. Our study demonstrated that patients with complicated infections, especially cellulitis or infections involving the leg, and presence of at least one of the SIRS criteria, excluding temperature alterations, were more likely to have been upgraded to the OU. Ninety-six-hour ED revisit or hospitalization was relatively uncommon with only $9.7 \%$ of the entire cohort experiencing this endpoint. This is likely a reflection of the overall low acuity of these patients. Few parameters were associated 
Table 2 Antimicrobial therapy by setting of care (emergency department versus observation unit)

\begin{tabular}{lccr}
\hline Antibiotic & Emergency department $(\boldsymbol{n}=\mathbf{2 1 9})$ & Observation unit $(\boldsymbol{n}=\mathbf{8 9})$ & $\boldsymbol{p}$ value \\
\hline Empiric IV therapy & $31(14.2)$ & $35(39.3)$ & $<0.001$ \\
Empiric vancomycin & $128(58.4)$ & $26(29.2)$ & $<0.001$ \\
Empiric clindamycin & $37(16.9)$ & $19(21.3)$ & 0.415 \\
Empiric ampicillin/sulbactam & & & \\
Discharge antibiotic & $19(8.7)$ & $12(13.4)$ & 0.214 \\
Amoxicillin/clavulanate & $41(18.7)$ & $7(7.8)$ & 0.049 \\
Cephalexin & $106(48.4)$ & $30(33.7)$ & 0.027 \\
Clindamycin & $8(3.7)$ & $1(1.1)$ & $0.455^{\mathrm{b}}$ \\
Doxycycline & $1(0.5)$ & $1(1.1)$ & $0.495^{\mathrm{b}}$ \\
Linezolid & $16(7.3)$ & $18(20.2)$ & 0.002 \\
None & $17(7.8)$ & $22(24.7)$ & $<0.001$ \\
TMP/SMX & $36(16.4)$ & $8(9.0)$ & 0.107 \\
Dual therapy & $\mathrm{a}$ & $1 V(10)$ \\
\hline
\end{tabular}

CAMRSA community acquired methicillin-resistant Staphylococcus aureus, IV intravenous, TMP/SMX trimethoprimsulfamethoxazole

${ }^{a}$ Coverage of CAMRSA and streptococcal species

${ }^{\mathrm{b}}$ Fisher's exact test

with 96-h ED revisit or hospitalization upon multivariable analysis. Of note, a history of recurring ABSSSIs as well as prior MRSA infection were linked to the primary outcome. One factor that has previously been shown to increase risk of outpatient failure is meeting SIRS criteria [21]. Based on this, the Infectious Diseases Society of America (IDSA) guidelines recommend initial inpatient management for patients with SIRS criteria. A study by Volz and colleagues found that patients with infections on their hands were three times more likely to require escalation of care [22]. Additionally, a white blood cell count $\geq 15,000 / \mathrm{mm}^{3}$ and female gender have been independently associated with failure to be successfully discharged from the OU [12]. The presence of fever (temperature $\geq 38^{\circ} \mathrm{C}$ ) was the most commonly reported predictor for requiring a hospital stay of $\geq 24 \mathrm{~h}$ or failure of $\mathrm{ED} / \mathrm{OU}$ status $[13,22]$. In our cohort, the presence of fever was found to be significantly linked to 96-h ED revisit/admission among those treated in the ED, likely a signal of requiring upgrade to the OU. Future research should aim to determine if these parameters can be generally applied to determine which patients are likely to require escalation of care to avoid subsequent ED revisits.

Severity of illness was evaluated using two scoring systems not commonly employed in the United States-the CREST/Eron Classification and the SEWS $[18,19]$. These systems were employed because there is currently not a commonly used or validated tool in the United States. Neither correlated with site of care, suggesting that the management decisions in the ED/OU setting for ABSSSI are likely based 
Table 3 Univariate analysis of 96 -h revisit or hospital admission

\begin{tabular}{|c|c|c|c|}
\hline Characteristics & $96 \mathrm{~h}$ revisit $(n=30)$ & No revisit $(n=278)$ & $p$ value \\
\hline ED setting of care & $23(76.7)$ & $19(70.5)$ & 0.479 \\
\hline CREST/Eron class II & $8(26.7)$ & $57(20.5)$ & 0.701 \\
\hline \multicolumn{4}{|l|}{ Comorbid conditions } \\
\hline Diabetes & $6(20.0)$ & $44(15.8)$ & 0.566 \\
\hline Prior ABSSSI & $12(40.0)$ & $53(19.1)$ & 0.008 \\
\hline Prior MRSA infection & $3(10.0)$ & $4(1.4)$ & $0.003^{\mathrm{a}}$ \\
\hline Morbid obesity & $6(20.0)$ & $34(12.2)$ & 0.250 \\
\hline Charlson comorbidity score (IQR) & $1.0(0.0-1.3)$ & $1.0(0.0-2.0)$ & 0.601 \\
\hline Surgical intervention & $12(40.0)$ & $137(49.3)$ & 0.345 \\
\hline Antibiotics 90 Days & $3(10.0)$ & $47(16.9)$ & 0.382 \\
\hline \multicolumn{4}{|l|}{ SIRS criteria on presentation } \\
\hline Body temp $>38$ or $<36$ & $11(36.7)$ & $28(20.9)$ & 0.049 \\
\hline $\mathrm{HR}>90$ beats $/ \mathrm{min}$ & $14(46.7)$ & $123(44.2)$ & 0.800 \\
\hline $\mathrm{RR}>20$ breaths $/ \mathrm{min}$ & $1(3.3)$ & $13(4.7)$ & $1.000^{\mathrm{a}}$ \\
\hline WBC $>12,000$ or $<4000$ & $4(13.3)$ & $26(9.4)$ & 0.485 \\
\hline \multicolumn{4}{|l|}{ Site of infection } \\
\hline Arm & $3(10.0)$ & $52(18.7)$ & $0.319^{\mathrm{a}}$ \\
\hline Leg & $7(23.3)$ & $54(19.4)$ & 0.631 \\
\hline Head/neck & $3(10.0)$ & $51(18.3)$ & 0.320 \\
\hline Buttocks/torso & $13(43.3)$ & $72(25.9)$ & 0.042 \\
\hline Hand & $1(3.3)$ & $30(10.8)$ & $0.197^{\mathrm{a}}$ \\
\hline Foot & $3(10.0)$ & $17(6.1)$ & $0.427^{\mathrm{a}}$ \\
\hline \multicolumn{4}{|l|}{ Type of ABSSSI } \\
\hline Complicated cellulitis & $3(10.0)$ & $35(12.6)$ & $0.682^{\mathrm{a}}$ \\
\hline Complicated abscess & $6(20.0)$ & $57(20.5)$ & 0.948 \\
\hline Uncomplicated cellulitis & $9(30.0)$ & $77(27.7)$ & 0.789 \\
\hline Uncomplicated abscess & $11(36.7)$ & $88(31.7)$ & 0.577 \\
\hline \multicolumn{4}{|l|}{ Empiric antibiotics } \\
\hline Ampicillin/sulbactam & $3(10.0)$ & $53(19.1)$ & $0.319^{\mathrm{a}}$ \\
\hline \multicolumn{4}{|l|}{ Discharge antibiotics } \\
\hline Combination therapy & $6(20.0)$ & $38(13.7)$ & 0.408 \\
\hline Tissue culture available & $6(20.0)$ & $49(17.6)$ & 0.802 \\
\hline
\end{tabular}

$A B S S S I$ acute bacterial skin and skin structure infection, CREST Clinical Resource Efficiency Support Team, $H R$ heart rate, $I Q R$ interquartile range, MRSA methicillin-resistant Staphylococcus aureus, $R R$ respiratory rate, $W B C$ white blood cell

${ }^{a}$ Fisher's exact test 
Table 4 Multivariable regression analysis of risk factors for 96-h ED revisit or hospital admission

\begin{tabular}{lllllll}
\hline Factor & Unadjusted OR & $\mathbf{9 5 \%}$ CI & $\boldsymbol{p}$ value & Adjusted OR & 95\% CI & $\boldsymbol{p}$ value \\
\hline Prior ABSSSI & 2.83 & $1.29-6.23$ & 0.013 & 2.38 & $1.26-6.35$ & 0.014 \\
Prior MRSA & 7.61 & $1.62-35.79$ & 0.020 & 4.38 & $0.80-24.20$ & 0.072 \\
Temperature $>38$ or $<36^{\circ} \mathrm{C}$ & 2.19 & $0.99-4.87$ & 0.061 & 2.20 & $0.97-5.00$ & 0.059 \\
Location Torso/Buttocks & 2.19 & $1.01-4.73$ & 0.042 & 2.36 & $1.07-5.20$ & 0.038 \\
\hline
\end{tabular}

ABSSSI acute bacterial skin and skin structure infection, $C I$ confidence interval, $O R$ odds ratio, MRSA methicillin-resistant Staphylococcus aureus

on factors not included in current criteria. Comorbid conditions requiring additional management and optimization may be influencing these decisions. Neither CREST/ Eron nor SEWS has been validated to determine ABSSSI management in $\mathrm{ED}$ versus OU specifically. Marwick and colleagues observed that $70 \%$ of patients in CREST/Eron Class II (normally recommended to treat as inpatient with IV antibiotics for up to $48 \mathrm{~h}$ ) could be treated on an outpatient basis [19]. In our study there was no significant difference in the amount of CREST/Eron Class II patients between ED and OU, supporting the previous finding. Physiological parameters within SEWS were better able to differentiate patients that were upgraded to the $\mathrm{OU}$, which also mirrors findings from Marwick and colleagues in their attempt to prospectively validate a modified CREST/Eron Classification system [23]. Higher CREST/Eron Class or SEWS were not associated with 96-h ED revisit/hospitalization. Even though severity scoring could potentially identify patients requiring observation for a period of up to $48 \mathrm{~h}$, our results suggest that the majority of patients, especially those with uncomplicated cellulitis or abscess, do not need this extended period of healthcare exposure. Aligned with data on outpatient parental antimicrobial therapy data (OPAT), use of OUs with or without subsequent OPAT can lead to reduced hospital costs, decreased healthcare exposure and risk of adverse events, and improve patient care [10, 24, 25].

Numerous investigations have examined the appropriateness of antibiotic prescribing for ABSSSIs in EDs, especially with the rising prevalence of CAMRSA [26-29]. Pallin and colleagues evaluated data form 2007-2010 NHAMCS using the quality measures of overuse, underuse, and misuse. Overuse was defined as use of any antibiotic for abscess patients with successful incision and drainage or use of CAMRSA agents in patients with cellulitis (no purulence). In our study, over $80 \%$ of patients with incision and drainage were prescribed antibiotics on discharge and over $60 \%$ of patients with cellulitis were prescribed an agent active against CAMRSA. Double coverage against both streptococcal spp. and CAMRSA was also a common occurrence, which increases the risk of adverse drug events and resistance [30]. These data support the need for improvement initiatives in prescribing patterns for ABSSSIs.

Diagnostics procedures were significantly different in patients treated in the ED versus $\mathrm{OU}$, which is not surprising based on the difference in time receiving care and specialty of the treating physicians. Routine blood cultures, drawn in over $50 \%$ of OU patients, have not been shown to improve clinical 
outcomes, and are not recommended routinely by the IDSA for the treatment of skin and skin structure infections [21, 31, 32]. The American Board of Internal Medicine developed the Choose Wisely Campaign to limit unnecessary tests and procedures in the treatment of various medical conditions [15]. ACEP, a partner organization, lists the use of antibiotics and cultures in uncomplicated abscesses after incision and drainage with medical follow-up among their top 10 unnecessary procedures and tests [33]. Our data demonstrate that in the ED over $60 \%$ of cultures were taken in uncomplicated abscesses. It is important to note, however, that due to the retrospective nature of the study we cannot assess if appropriate outpatient follow-up was available or if the cultures and susceptibility data from the ED was conveyed to each applicable patient. Our findings are similar to Jenkins and colleagues wherein arguably avoidable healthcare resources were employed for diagnostic testing [26]. Among the 322 inpatients included in their analysis, $47-58 \%$ of patients had blood cultures drawn, of which 13 patients were bacteremic. Radiological data were also commonly used to rule/out deeper sites of infection. Among patients with cellulitis, $94 \%$ received a plain film radiograph, with a positive yield of only $1 \%$. It is important to note, however, that Jenkins and colleagues studied hospitalized patients with ABSSSSIs where our cohort was entirely treated in the ED/OU setting.

\section{Limitations}

There are several limitations to this study that should be addressed. First, the study was retrospective in nature, and thus relied on proper documentation within electronic medical charts. This may be especially important for history of MRSA and size of the lesion. The study was also single centered, which may limit the external generalizability of the results. It is unclear what differences in practice patterns or patient acuity may exist among patients treated for ABSSSIs at other institutions. In addition, the fact that DMC is only one of several large academic medical centers within the Detroit metropolitan area makes it likely that some ED revisits were missed. Much like the study with Pallin and colleagues, patients with incision and drainage may have been prescribed outpatient antibiotics due to a large area of induration around the lesion, thus falsely increasing the cases in our quality measure of overuse [28]. The study consists of patients treated with IV antibiotics only, but the decision to administer IV versus orally is also highly subjective and may contribute to our inability to discern differences between settings of care or 96-h ED revisit/hospitalization. Lastly, resource utilization for follow-up in ambulatory care clinics was not evaluated.

\section{CONCLUSION}

Data regarding the decision strategies and processes of care in patients with ABSSSIs with low acuity, especially those treated in OUs, are relatively unreported. This study demonstrated the subjective nature of determining patient level of care as well as the varied diagnostic tests/procedures and prescribing patterns for patients discharged with ABSSSIs. Resources utilized in the diagnosis and management of these lower acuity ABSSSIs should be critically evaluated to determine necessity and benefit to the patient. Additionally, few differences in patient presentation were significantly linked to the decision to escalate care to OU status. 
Across both settings, ED revisit or hospitalization within $96 \mathrm{~h}$ was infrequent; suggesting that short observation and less than $24 \mathrm{~h}$ of IV therapy followed by oral therapy is appropriate management for many ABSSSIs, particularly in patients with no prior history of infection. Several considerations, such as past history of ABSSSI, temperature alterations at presentation, or certain locations of ABSSSIs may be important indicators for need to escalate care. There is, however, a need for more evidence-driven guidance of patient disposition and resources necessary to optimize patient outcomes while decreasing wasteful use of finite and costly resources.

\section{ACKNOWLEDGMENTS}

No funding or sponsorship was received for this study or publication of this article. KCC, SLD, and MJR conceived and designed the study. SLD and MJR supervised research conduct and medical chart review. KCC, AML, TBP, and MGJ undertook medical chart review and data collection. KCC managed and analyzed the data. KCC drafted the manuscript and all authors contributed substantially to its revision. All named authors meet the International Committee of Medical Journal Editors (ICMJE) criteria for authorship for this manuscript, take responsibility for the integrity of the work as a whole, and have given final approval to the version to be published. MJR takes responsibility for the paper as a whole.

Conflict of interest. MJR has received research support, consulted or participated on speaker bureaus for Cepheid, Cubist, Durata, Forest, Theravance, Trius, and is partially supported by the NIH. SLD has received grant support from Cubist and Forest and served as a consultant for Forest, Durata, Premier, and Pfizer. KCC, AML, TBP and MGJ have no conflicts of interest to disclose.

Ethical standard. This was an institutional review board approved, retrospective, observational study at an urban, academic medical center. All procedures followed were in accordance with the ethical standards of the responsible committee on human experimentation (institutional and national) and with the Helsinki Declaration of 1975 , as revised in 2000. Informed consent was waived by Wayne State University institutional review board.

Open Access. This article is distributed under the terms of the Creative Commons Attribution Noncommercial License which permits any noncommercial use, distribution, and reproduction in any medium, provided the original author(s) and the source are credited.

\section{REFERENCES}

1. Dryden MS. Complicated skin and soft tissue infection. J Antimicrob Chemother. 2010;65(Suppl 3):iii35-44.

2. Edelsberg J, Taneja C, Zervos M, Haque N, Moore C, Reyes $\mathrm{K}$, et al. Trends in US hospital admissions for skin and soft tissue infections. Emerg Infect Dis. 2009;15(9):1516-8.

3. National Hospital Ambulatory Medical Care Survey: 2010 Emergency Department Summary Tables. 2010. http://www.cdc.gov/nchs/data/ahcd/ nhamcs_emergency/2010_ed_web_tables.pdf. Accessed on Sept 14, 2014.

4. Hersh AL, Chambers HF, Maselli JH, Gonzales R. National trends in ambulatory visits and antibiotic prescribing for skin and soft-tissue infections. Arch Intern Med. 2008;168(14):1585-91.

5. Pallin DJ, Egan DJ, Pelletier AJ, Espinola JA, Hooper DC, Camargo CA Jr. Increased US emergency department visits for skin and soft tissue 
infections, and changes in antibiotic choices, during the emergence of community-associated methicillin-resistant Staphylococcus aureus. Ann Emerg Med. 2008;51(3):291-8.

6. Koerner R, Johnson AP. Changes in the classification and management of skin and soft tissue infections. J Antimicrob Chemother. 2011;66(2):232-4.

7. Peterson D, McLeod S, Woolfrey K, McRae A. Predictors of failure of empiric outpatient antibiotic therapy in emergency department patients with uncomplicated cellulitis. Acad Emerg Med. 2014;21(5):526-31.

8. Murray H, Stiell I, Wells G. Treatment failure in emergency department patients with cellulitis. CJEM. 2005;7(4):228-34.

9. Berger A, Oster G, Edelsberg J, Huang X, Weber DJ. Initial treatment failure in patients with complicated skin and skin structure infections. Surg Infect. 2013;14(3):304-12.

10. American College of Emergency Physicians. State of the art: observation units in the emergency department [Policy Resource and Education Paper]. 2011. http://www.acep.org/ClinicalPractice-Management/Emergency-Department-Obs ervation-Services/. Accessed Feb 1, 2015.

11. Sheehy AM, Graf B, Gangireddy S, Hoffman R, Ehlenbach M, Heidke C, et al. Hospitalized but not admitted: characteristics of patients with "observation status" at an academic medical center. JAMA Intern Med. 2013;173(21):1991-8.

12. Schrock JW, Laskey S, Cydulka RK. Predicting observation unit treatment failures in patients with skin and soft tissue infections. Int J Emerg Med. 2008;1(2):85-90.

13. Sabbaj A, Jensen B, Browning MA, John Ma O, Newgard CD. Soft tissue infections and emergency department disposition: predicting the need for inpatient admission. Acad Emerg Med. 2009;16(12):1290-7.

14. Crenshaw LA, Lindsell CJ, Storrow AB, Lyons MS. An evaluation of emergency physician selection of observation unit patients. Am J Emerg Med. 2006;24(3):271-9.

15. Foundation American Board of Internal Medicine. Choose wisely. 2015. http://www.choosingwisely. org/. Accessed Feb 17, 2015.

16. Food and Drug Administration (FDA) Guidance for Industry: Acute Bacterial Skin and Skin Structure Infections: Food and Drug Administration; 2013.
http://www.fda.gov/downloads/Drugs/.../Guidanc es/ucm071185.pdf. Accessed Sept 18, 2014.

17. Food and Drug Administration (FDA) Guidance for industry: uncomplicated and complicated skin and skin structure infections: developing drugs for treatment. 1998. http://www.fda.gov/ohrms/dockets/ 98fr/2566dft.pdf. Accessed Feb 10, 2014.

18. Clinical Resource Efficiency Team (CREST). Guidelines on the management of cellulitis in adults. 2005. http://www.acutemed.co.uk/docs/Cellulitis guidelines,CREST,05.pdf. Accessed Sept 14, 2014.

19. Marwick C, Broomhall J, McCowan C, Phillips G, Gonzalez-McQuire S, Akhras K, et al. Severity assessment of skin and soft tissue infections: cohort study of management and outcomes for hospitalized patients. J Antimicrob Chemother. 2011;66(2):387-97.

20. Dumkow LE, Kenney RM, MacDonald NC, Carreno JJ, Malhotra MK, Davis SL. Impact of a multidisciplinary culture follow-up program of antimicrobial therapy in the emergency department. Infect Dis Ther. 2014;3(1):45-53.

21. Stevens DL, Bisno AL, Chambers HF, Dellinger EP, Goldstein EJ, Gorbach SL, et al. Practice guidelines for the diagnosis and management of skin and soft tissue infections: 2014 update by the infectious diseases society of America. Clin Infect Dis. 2014;59(2):147-59.

22. Volz KA, Canham L, Kaplan E, Sanchez LD, Shapiro NI, Grossman SA. Identifying patients with cellulitis who are likely to require inpatient admission after a stay in an ED observation unit. Am J Emerg Med. 2013;31(2):360-4.

23. Marwick C, Rae N, Irvine N, Davey P. Prospective study of severity assessment and management of acute medical admissions with skin and soft tissue infection. J Antimicrob Chemother. 2012;67(4):1016-9.

24. Jones GR, Cumming DV, Honeywell G, Ball R, Sanderson F, Seaton RA, et al. How is income generated by outpatient parenteral antibiotic treatment (OPAT) in the UK? Analysis of payment tariffs for cellulitis. J Antimicrob Chemother. 2015;70(4):1236-40.

25. Nazarko L. Avoiding admission and facilitating early discharge through OPAT. Br J Nurs. 2014;23(14):S30-6.

26. Jenkins TC, Sabel AL, Sarcone EE, Price CS, Mehler PS, Burman WJ. Skin and soft-tissue infections requiring hospitalization at an academic medical center: opportunities for antimicrobial stewardship. Clin Infect Dis. 2010;51(8):895-903. 
27. Meddles-Torres $\mathrm{C}, \mathrm{Hu} \mathrm{S}$, Jurgens $\mathrm{C}$. Changes in prescriptive practices in skin and soft tissue infections associated with the increased occurrence of community acquired methicillin resistant Staphylococcus aureus. J Infect Public Health. 2013;6(6):423-30.

28. Pallin DJ, Camargo CA Jr, Schuur JD. Skin infections and antibiotic stewardship: analysis of emergency department prescribing practices, 2007-2010. West J Emerg Med. 2014;15(3):282-9.

29. Mistry RD, Shapiro DJ, Goyal MK, Zaoutis TE, Gerber JS, Liu C, et al. Clinical management of skin and soft tissue infections in the U.S. emergency departments. West J Emerg Med. 2014;15(4):491-8.

30. Walraven CJ, Lingenfelter E, Rollo J, Madsen T, Alexander DP. Diagnostic and therapeutic evaluation of community-acquired methicillin- resistant Staphylococcus aureus (MRSA) skin and soft tissue infections in the emergency department. J Emerg Med. 2012;42(4):392-9.

31. Perl B, Gottehrer NP, Raveh D, Schlesinger Y, Rudensky B, Yinnon AM. Cost-effectiveness of blood cultures for adult patients with cellulitis. Clin Infect Dis. 1999;29(6):1483-8.

32. Paolo WF, Poreda AR, Grant W, Scordino D, Wojcik $S$. Blood culture results do not affect treatment in complicated cellulitis. J Emerg Med. 2013;45(2):163-7.

33. American College of Emergency Physicians (ACEP). Five things physicians and patients should question. http://www.choosingwisely.org/doctorpatient-lists/american-college-of-emergency-physici ans/. Accessed Feb 17, 2015. 